Christine Kettle*, DPT, Lauren McKay, DO, Anthony M. Cianciolo, DO, Stephen M. Kareha, DPT, ATC, PhD and Cara E. Ruggeri, DO

\title{
A novel movement system screen for primary care providers: a multisite, observational study
}

https://doi.org/10.1515/jom-2021-0185

Received July 17, 2021; accepted November 8, 2021;

published online January 10, 2022

\begin{abstract}
Context: Movement of the human body is essential for the interaction of an individual within their environment and contributes to both physical and emotional quality of life. Movement system disorders (MSDs) are kinesiopathologic conditions that result from either altered movement patterns, trauma, or pathology. A screening tool may facilitate earlier diagnosis and treatment of acute MSDs. This tool could prevent progression to chronic conditions, leading to better patient outcomes and quality of life.
\end{abstract}

Objectives: Our study evaluated whether a screening tool would be able to accurately screen individuals for MSDs, explore comorbidities that may predict the prevalence of MSDs, and identify why people do not discuss these problems with their primary care provider (PCP).

Methods: A multisite, observational study in a primary care setting. Data were analyzed to determine the psychometric properties of the screening question. Logistic regression was performed to explore the relationship of

\footnotetext{
*Corresponding author: Christine Kettle, DPT, Physical Therapy at St. Luke's, St. Luke's University Health Network, Bethlehem, PA, USA; and Physical Therapy at St. Luke's, St. Luke's University Health Network, 501 Cetronia Road, Allentown, PA 18104-9569, USA,

E-mail: christine.kettle@sluhn.org. https://orcid.org/0000-00030631-9795
}

Lauren McKay, DO, Department of Internal Medicine, St. Luke's University Health Network, Bethlehem, PA, USA

Anthony M. Cianciolo, DO, Internal Medicine, St. Luke's University Health Network, Bethlehem, PA, USA

Stephen M. Kareha, DPT, ATC, PhD, Physical Therapy at St. Luke's, St. Luke's University Health Network, Bethlehem, PA, USA; and Department of Physical Therapy, DeSales University, Center Valley, PA, USA. https://orcid.org/0000-0002-7392-535X

Cara E. Ruggeri, DO, Department of Internal Medicine, St. Luke's University Health Network, Bethlehem, PA, USA; and Clinical Associate Professor (Adjunct), Lewis Katz School of Medicine, Temple University School of Medicine, Philadelphia, PA, USA comorbidities with MSDs. Thematic analysis was performed to explore why patients do not discuss these issues with their PCP.

Results: The point prevalence of MSDs was determined to be $78 \%$. The sensitivity of the screening question was determined to be good (70\%). Arthritis, obesity, sleep disorders, and gastroesophageal reflux disease (GERD) were significant predictors for an MSD. Thematic analysis regarding why patients do not discuss the MSD with their physician revealed: (1) the perceived lack of importance of the problem; (2) the lack of access to healthcare, and (3) the acuity of the problem.

Conclusions: Screening for an MSD and associated comorbidities could prevent the transition of acute conditions to chronic conditions. If PCPs can identify predictors and factors associated with an MSD, they may be able to screen for MSDs more effectively. Earlier identification of MSDs may facilitate earlier treatment and prevent costs associated with resulting chronic disorders and persistent pain and disability.

Keywords: chronic; disability; movement system; movement system disorder; primary care; screening tool.

Movement is a complicated action; it is integral to human behavior, essential to physical and emotional quality of life, and is affected by social, environmental, and personal factors [1]. Unsurprisingly, movement involves multiple biological systems [2, 3]. The movement system is a conceptual framework unifying the aspects of the musculoskeletal, cardiovascular, pulmonary, nervous, integumentary, and endocrine systems, which generate and maintain movement at all levels of bodily function [1, 4]. Therefore, movement system disorders (MSDs) are kinesiopathologic conditions that result from altered movement patterns (e.g., poor scapulohumeral rhythm resulting in difficulty reaching overhead), trauma (e.g., injury resulting from a fall causing difficulty walking), or pathology (e.g., Parkinson's disease resulting in difficulty initiating movement) [1]. Due to the wide and varied spectrum of MSDs, this makes kinesiopathologic conditions of economic, medical, and scientific significance [5]. 
MSDs likely have a significant burden on the United States healthcare system and contribute greatly to healthcare spending [6]. Some of the most common MSDs are gait dysfunction [7], low back pain [8], ankle injury [9], and pelvic floor disorders [10]. Although specific data on all MSDs are not available, previous research by the U.S. Department of Health and Human Services on musculoskeletal MSDs from 2014 found that based upon a sample of 6,045 people, the estimated annual cost per person was \$9,554 (SD, \$315) [5, 11]. Based upon an estimated population of 65 million people with a musculoskeletal MSD, the estimated annual costs of musculoskeletal MSDs is estimated at $\$ 626$ million [11]. Furthermore, this high cost is compounded by many patients not reporting an MSD until the patient personally views the problem as urgent, which may result in more extensive imaging and treatments [10]. Because patients may not initially view MSDs as problematic, they may not report them to their provider unless screened appropriately. If barriers to movement and physical activity exist, screening enables a patient to be identified early to prevent and mitigate the transition from acute to chronic disorders and the associated costs. A specific screening tool may also be useful because studies have found that many medical students [12, 13], residents [14], and attending physicians [14] in nonorthopedic specialties think that they have inadequate musculoskeletal knowledge. Unfortunately, the only reliable method to screen for an MSD is to ask about every possible movement action and function [15]. This would include over 40 additional items beyond a typical primary care visit and thus is unrealistic given the time constraints and requirements in contemporary family practice.

A common theme to help curtail costs is to implement screening measures for diseases with the greatest impact on the health and well-being of patients and, financially, on the healthcare system. One such mechanism of standardizing screening measures has been through Meritbased Incentive Payment System (MIPS) [16]. This system results in either a bonus payment for performance of specific screening measures or financial penalty for not performing and reporting specific screening measures [16].

Two widely utilized measures are for obesity and fall risk. With appropriate screening, the U.S. Preventative Services Task Force has found sufficient evidence to support the benefits of early detection of obesity with appropriate referral and intervention to minimize associated costs and complications [17]. Additionally, fall risk assessment is another value-based screening tool for primary care providers (PCPs) [18]. Data have shown a 25\% reduction in falls with appropriate screening and referral [18]. Thus, we postulate that if a similar merit program and screening tool are implemented for MSDs, the associated healthcare costs and treatment time may be decreased while the quality of life may be increased.

In an effort to establish an efficient screening method for MSDs, a single-item screening tool was developed by two medical researchers and utilized in a pilot study [15]. The intent of this screening question was to minimize the number of questions that a PCP would need to ask to rule out an MSD [15]. Additionally, a content expert group, including four PCPs, two physical therapists, one statistician, and one bilingual research scientist, identified 40 functional questions that span all body systems related to human movement, which were identified as a gold standard for establishing the presence of an MSD [15]. Based upon the pilot study results and feedback from the content expert group, the screening tool [15] was modified for the current study.

This study sought to: (1) evaluate whether a single-item screening tool utilized in the primary care setting would be able to accurately screen individuals for MSDs; (2) explore comorbidities that may predict a higher risk for MSDs; and (3) identify why patients delay discussion of these problems with their PCPs.

\section{Methods}

We conducted an observational study of patients arriving for a primary care visit in a regional health network. Ethical approval was obtained through St. Luke's University Health Network's Institutional Review Board as protocol SLQI 2017-80. The authors received no financial support for the research, authorship, and/or publication of this article.

\section{Setting and participants}

A convenience sample of consecutive patients arriving for a primary care visit was recruited from four practices in Pennsylvania and New Jersey over a period of 14 months, January 2018 through March 2019. The exclusion criteria were under age 18 years and lack of literacy in English or Spanish. All other patients presenting for an office visit for any reason were eligible for inclusion. Consent was provided verbally prior to completing the survey.

\section{Measures}

The subjects completed the survey while waiting for the provider. Participants were asked to fill out a one-page survey (Supplementary Appendices). After responding to the single-item screening question ("Do you have pain or difficulty when starting or performing any physical activity?"), participants were then encouraged to complete the remainder of the survey. An MSD was identified if the participant selected any activity or body region with which they were having pain or difficulty with movement (Figure 1 and Table 1). Survey items were 


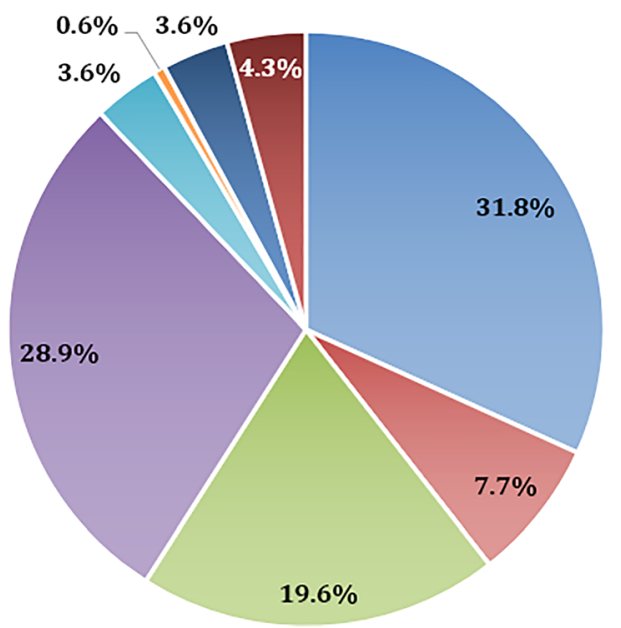

- Back/Neck/Spine

" Head/Face/Jaw

- Shoulder/Elbow/Hand

= Hip/Knee/Ankle/Foot

n Neurologic Disorder

Concussion

* Incontinence/Pelvic Health

n Other

Figure 1: Percentage of movement system disorders (MSDs) by body region.

Table 1: Number of participants with pain or difficulty during these activities.

\begin{tabular}{lrlr}
\hline Activity & $\mathbf{n , \%}$ & Activity & $\mathbf{n , \%}$ \\
\hline Balance & $190(29.9 \%)$ & Sexual activity & $76(11.9 \%)$ \\
Bending & $284(44.7 \%)$ & Shopping & $73(11.5 \%)$ \\
Chewing & $20(3.1 \%)$ & Sitting & $128(20.1 \%)$ \\
Concentrating & $118(18.6 \%)$ & Sleeping & $233(36.6 \%)$ \\
Cooking & $76(11.9 \%)$ & Squatting & $187(29.4 \%)$ \\
Dressing & $107(16.8 \%)$ & Stairs & $263(41.4 \%)$ \\
Driving & $66(10.4 \%)$ & Standing & $197(31.0 \%)$ \\
Eating/drinking & $40(6.3 \%)$ & Talking & $14(2.2 \%)$ \\
Lifting/carrying & $280(44.0 \%)$ & Throwing & $78(12.3 \%)$ \\
Looking up or turning & $122(19.2 \%)$ & Toileting & $40(6.3 \%)$ \\
neck & & & \\
Pushing/pulling & $164(25.8 \%)$ & Urinating & $39(6.1 \%)$ \\
Reaching & $132(20.8 \%)$ & Walking & $220(34.6 \%)$ \\
Running & $220(34.6 \%)$ & Other & $25(3.9 \%)$ \\
\hline
\end{tabular}

included to help understand patient perspectives regarding speaking to their PCP about MSDs. The electronic medical record for each of the participants was reviewed for comorbidities found to be correlated with increased healthcare spending $[16,17,19-26]$. Study data were collected and managed using REDCap (Research Electronic Data Capture, Version 6.4.6.- (C) 2017, Vanderbilt University) [11, 27].

\section{Statistical analysis}

Data were analyzed using SAS 9.4 (Cary, NC). A priori power analysis was used to calculate sample size. The parameters for the analysis were set as 0.80 , and a type I error rate $(\alpha)$ of 0.05 was utilized to determine a target sample size of 998 participants. Descriptive statistics were analyzed for demographic information and point prevalence of MSDs, diagnostic accuracy was determined for the screening question, and logistic regression was performed to determine the predictive validity of MSDs utilizing IBM SPSS (version 25).

Demographic variables, age, and gender were summarized with means and standard deviations for continuous variables and
Table 2: Results showing sensitivity, specificity, PPV, and NPV for a single-item screening tool in accurately detecting MSD.

\begin{tabular}{llll}
\hline & MSD present (+) & MSD not present (-) & $\begin{array}{l}\text { Predictive } \\
\text { value }\end{array}$ \\
\hline $\begin{array}{l}\text { Screening } \\
\text { question (+) }\end{array}$ & 478 & 0 & PPV 1.00 \\
$\begin{array}{l}\text { Screening } \\
\text { question (-) }\end{array}$ & 202 & 257 & NPV 0.56 \\
& Sensitivity 0.70 & Specificity 1.00 & $n=937$ \\
\hline
\end{tabular}

MSD, movement system disorder; NPV, negative predictive value; PPV, positive predictive value.

frequencies for nominal variables. A multivariate logistic regression model was conducted to identify comorbidities theoretically of clinical importance. This type of model building is utilized to best predict outcomes based on a series of predictor variables [28]. Associations between comorbidities as predictors of MSD were examined using a multivariate logistic regression model.

Inductive thematic analysis was performed to explore why patients delay bringing these issues to the attention of their PCP [29]. We utilized a constant comparative method to interpret data and identified common themes utilizing an open-coding method [29].

\section{Results}

\section{Screening tool accuracy}

A total of 1,007 participants, comprised of 286 males (28.5\%), 699 females (69.4\%), and 22 unidentified gender (2.2\%), were included in this study. The participants were aged between 18 and 100 years, with the mean age of 49.0 years (SD, 16.4) were included. MSDs were found to have a point prevalence of $78 \%$. Table 2 shows the correlated results between the single item-screening tool and the current gold standard (asking all 
Table 3: $A O R$ and $p$-value for covariates predictive of $\operatorname{MSD}[13,15$,

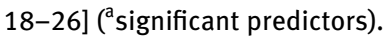

\begin{tabular}{|c|c|c|}
\hline Covariate & AOR $(95 \% \mathrm{Cl})$ & p-Value \\
\hline Arthritis $^{a}$ & $2.51(1.42-4.42)$ & 0.002 \\
\hline Asthma & $1.19(0.77-1.87)$ & 0.43 \\
\hline $\mathrm{BMI}>30^{\mathrm{a}}$ & $1.40(1.04-1.89)$ & 0.03 \\
\hline Depression or anxiety & $1.13(0.82-1.56)$ & 0.46 \\
\hline Dyslipidemia/hyperlipidemia & $0.87(0.61-1.23)$ & 0.43 \\
\hline GERD $^{\mathrm{a}}$ & $2.55(1.63-3.99)$ & $<0.0001$ \\
\hline Hypertension & $1.18(0.83-1.68)$ & 0.35 \\
\hline Sleep disorder ${ }^{a}$ & $1.68(1.02-2.78)$ & 0.04 \\
\hline Somatic syndromes & $\begin{array}{l}\mathrm{N} / \mathrm{A} \text { (quasi separation } \\
\text { of data points, meaning } \\
\text { there were no somatic } \\
\text { syndromes among } \\
\text { patients without a } \\
\text { primary MSD) }\end{array}$ & $\mathrm{N} / \mathrm{A}$ \\
\hline
\end{tabular}

${ }^{\text {a }}$ Pearson chi square $p$-value $=0.75 ; c$-statistic $=70$. AOR, adjusted odds ratio; $\mathrm{BMI}$, body mass index; $\mathrm{Cl}$, confidence interval; GERD, gastroesophageal reflux disease.

40 items on the questionnaire). True positives were considered as those who answered "yes" to the screening question and identified a specific movement(s) as difficult; true negatives were considered those who answered "no" to the screening question and did not select a specific movement(s) as difficult. False positives were those who answered "yes" to the screening question but did not identify a specific movement(s) as difficult; false negatives were considered as those who answered "no" to the screening question but identified a movement(s) as difficult. The single itemscreening tool was found to have a sensitivity of $70 \%$, specificity of $100 \%$, a positive predictive value (PPV) of $100 \%$, and a negative predictive value (NPV) of $56 \%$.

\section{Predictors of MSD}

Of those 1,007 participants, 22 were excluded from the subsequent predictive analysis due to not providing enough legible identifying information to allow the researchers to review the medical chart. The direct multivariate logistic regression performed on the remaining 985 participants $(699$ females, 286 males; mean age 48.3 years, SD 17.2) identified the following covariates based on statistically significant bivariate results $(\mathrm{p}<0.020)$ and/or their clinical importance: arthritis [25], asthma [19], body mass index (BMI) >30 [25], depression or anxiety [26], dyslipidemia/hyperlipidemia [25], diabetes [25], gastroesophageal reflux disease (GERD) [22, 23], hypertension [24], sleep disorder [20, 21], and somatic syndromes [20].
Prior to model building, diagnostic assessment revealed that diabetes represented the sole likely confounder, based on a change in other covariates' parameter estimates of $<10 \%$ following its exclusion from the model; therefore, we did not include it in our final model [28]. Regarding outliers and/or influential data points, only 28/1,007 patients (2.8\%) had suggestive covariate patterns; given this small number, we decided to retain these patients in the final model [28].

We report adjusted odds ratios (AORs) and 95\% confidence intervals (CIs), with $\mathrm{p}<0.05$ denoting significance in the final model [28]. As shown in Table 3, arthritis $(p=0.002)$, BMI $>30(p=0.03)$, GERD $(p<0.0001)$, and sleep disorder $(\mathrm{p}=0.04)$ independently and significantly predicted having an MSD [28].

\section{Patient reporting}

Although a large percentage of participants in this study reported an MSD to their PCP, 25.3\% ( $n=255)$ of participants had not spoken to their PCP about their MSD. Utilizing an open-coding method, common themes from the list of predetermined responses and "other" comments (Supplementary Appendices A and B) were formed into categories. Participants identified three common reasons for why they did not discuss MSDs with their PCP. These themes were classified as: 1) having perceived lack of importance of the issue $(56.0 \%)$; 2) lack of access to healthcare $(13.8 \%)$; and 3) the MSD was an acute issue (10.1\%). Additionally, $1.6 \%$ of participants identified that they were not discussing it with their PCP because they were already being treated for their MSD.

\section{Perceived lack of importance}

Participants identified the primary reasons as to why patients are not discussing MSDs with their PCP as "I don't think anything can be done about it," "The problem is not important enough to me," and "I have other health issues that are more important." Several patients commented that their symptoms were "not that bad yet." One participant even mentioned that her symptom "hasn't affected ADLs or quality of life at this point."

\section{Lack of access to healthcare}

Another common theme found among participants was lack of access to healthcare. Related responses included "no physician until today" and "haven't had a doctor to talk to." One person mentioned that they were "not sure where to start." 


\section{Acute onset}

When asked about why they do not discuss MSDs with their PCP, 18 participants identified their issue being of an acute onset and they were there to discuss the MSD that day. For example, patients wrote that the issue "just started" or "here to talk about it today."

In contrast, several participants identified that they are not having these discussions because they are already being treated for the issue. Nevertheless, these themes elucidate why patients may wait until symptoms worsen before seeking help, which leads to costly and more drastic intervention.

\section{Discussion}

\section{Screening tool accuracy}

Clear health benefits for effective screening, referral, and implementation of intervention has been shown in people with obesity [30] and falls [18, 31]. Comparably, a brief and reliable screening tool for an MSD, as demonstrated in this study, can lead to appropriate onsite osteopathic manipulative therapy (OMT) and/or referral and intervention by a movement specialist, such as a physical therapist, which may reduce costs associated with chronic MSD. Interventions provided by both osteopathic physicians (e.g., OMT) and physical therapists (e.g., movement coordination training, biofeedback, soft tissue or joint manipulation, pain neuroscience education, strength training, endurance training, and wound management) can be more cost-effective in the treatment of chronic MSD compared to alternative medical treatments, such as medications (e.g., opioids) and surgery $[32,33]$. Importantly, osteopathic physicians skilled in OMT have a unique opportunity to employ techniques such as soft tissue treatments, counterstrain, muscle energy, high-velocity low-amplitude techniques, and balanced ligamentous maneuvers as point-of-care treatment for an array of musculoskeletal conditions while the patient is in the office encounter [33-35]. Due to time constraints in a single PCP office visit, a screen for an MSD will assist in bringing the MSD to the physician's attention. OMT can then be initiated at point of care, and if not possible at point of care or if further intervention is needed, the PCP can refer for consultation with a physical therapist.

\section{Predictors of MSDs}

Identifying a potential MSD early is essential for administering appropriate interventions (e.g., nonpharmacologic, pharmacologic, and surgical) and referring to the appropriate provider to mitigate rising healthcare costs [7-10, 36]. As our study demonstrates, four covariates could predict which patients should be initially screened for an MSD: obesity, arthritis, sleep disorders, and GERD (Table 3) $[13,15,16,19-26]$.

Obesity, and more specifically central adiposity, is a risk factor for osteoarthritis, which is frequently implicated as a cause of decreased mobility resulting in an MSD [37]. The link between osteoarthritis and MSDs may be further compounded due to the resulting decreased range of motion, muscle mass, and mobility leading to sedentary lifestyles [38].

The correlation between sleep disorders and MSDs was not surprising given the fact that sleep and pain thresholds have been extensively researched over the past few decades. Sleep deprivation and comorbid sleep problems are associated with altered pain processing [39], central sensitization, and generalized hyperalgesia [20]. Consequently, chronic sleep deprivation can also lead to chronic inactivity, which in turn creates an increased risk of MSD [21].

One of the most interesting findings in this study was a strong correlation between GERD and MSD. Although it has been reported that GERD may be overdiagnosed [22], and the use of proton pump inhibitors is excessive [23], an astounding finding from this study is that when GERD is not present, the likelihood of MSD is also lower. Furthermore, post hoc analysis of this data showed that there was no significant correlation between PPI use and MSD.

A few hypotheses exist for this correlation between GERD and MSD. It is known that GERD may exacerbate asthma, cough, and aspiration, which have the potential to limit one's activity level, leading to a more sedentary lifestyle [40]. Additionally, facilitation to spinal segments from visceral irritation in GERD can lead to somatic dysfunction in the thoracic spine, creating muscle tension, tenderness, asymmetry, and restriction of motion [41]. It is also possible that similar causative factors exist for GERD and MSD, resulting in both occurring due to potential anatomic and parasympathetic innervation overlap, specifically from the vagus nerve [41]. Lastly, the link between GERD and psychological health, specifically disorders of depression and anxiety, may also pose as a barrier for patients to remain in an active lifestyle [42]. 
Further research is needed to validate these correlations, yet if PCPs are screening for these conditions and MSD, referrals and treatment can be performed sooner, thus improving quality and cost-effective care, as shown by BMI and fall risk screening [17, 18]. This may be accomplished by earlier detection of MSD in patients with specific comorbidities, point-of-care OMT, and early consultation and treatment by physical therapists.

\section{Patient reporting}

Despite the frequency of somatic complaints and hallowed patient-physician relationship, many patients may still not feel comfortable sharing complaints consistent with MSD. Although cultural, educational, and socioeconomic factors likely contribute to the undersharing of MSD, in a study of 133 women, Steihaug et al. [36] found that patients "felt rejected with comments" that undermined their "experience of not being believed or understood [leading] to doubts regarding their own capacity to judge a situation." [36] In the same study, women who reported suffering with chronic pain reported lower self-esteem and shameful feelings leading to social isolation [36]. Additionally, in qualitative interviews from two studies totaling 100 patients presenting to emergency departments for nonurgent problems, participants discussed concerns that "their condition was too serious for the PCP, or that the ED provided more comprehensive services," or more simply, "it's urgent because it hurts" and "I'm afraid my condition is serious" [43, 44]. Therefore, the acuity of pain and lack of understanding of the MSD leads the public to seek care outside the primary care office for perceived rapidity of resolution for pain control and medical questions. Thus, an in-office screening tool may facilitate more focused, preventative, and socially "successful" visits because treatment, education, and understanding are managed simultaneously by both physicians and patients in a timely, nonurgent, and comprehensive manner.

Although not assessed in this study, physician comfort and time constraints may play a role in why MSDs may be overlooked. PCPs can be first-line to acknowledge MSD. Addressing MSD, when endorsed by patients, may be met with varying degrees of physician comfort and expertise. Not only can time constraints be a significant factor in appropriately screening for, examining, diagnosing, and treating MSD, but also competency and expertise likely vary widely. To address the competency and expertise gaps, the United States Bone and Joint Initiative in 2010 required medical training to incorporate musculoskeletal coursework into medical school training [12]. Additionally, the osteopathic philosophy and approach to patient care is critical to consider and expand upon for MSDs. In one study of 243 surveyed medical students, $57.1 \%$ of osteopathic students felt their musculoskeletal curriculum was adequate vs. $26.8 \%$ of allopathic students [13]. With an osteopathic approach and training, physicians acknowledge that the body is a unit, that there are a multitude of systems contributing to dysfunction, and also to healing, because the body is capable of self-regulatory mechanisms [13]. MSDs exemplify the tenet that structure follows function because the dysfunction creates further structural changes, potentially in skin, soft tissues, muscle tonicity, and skeletal alignment and movement planes [13]. By the same token, osteopathic treatment can alleviate or minimize pain, swelling, difficulty moving, or overall dysfunction of the body [13]. Therefore, MSDs and their treatment exemplify and easily incorporate the tenets of osteopathy, for which point-of-care OMT may be further enhanced with screening.

Additional limitations to this study include data were collected using a nonprobability strategy of convenience sampling, the study is only from four sites in a single regional health network, the study only included English and Spanish therefore excluding a minority of the national population, and further direct interviews were not performed with participants or their PCPs to further assess why MSDs were not discussed. In addition, no data were collected regarding how to best manage the MSDs found during this study.

Although not assessed in this study, screening for MSDs may improve patient care and decrease the overall cost of care $[8-10,45]$. Future studies should be aimed at evaluating whether patients who are screened and referred for consultation utilize fewer long-term interventions, such as the use of analgesics and opioids. Furthermore, future studies should compare the duration of treatment, cost of medical care, and functional ability in individuals screened for MSDs. Lastly, future studies should investigate what barriers may limit a PCP from initiating a discussion about MSDs.

\section{Conclusions}

MSD and associated comorbidities may not be captured by PCPs and therefore may lead to the transition of acute conditions to chronic problems. If PCPs can identify predictors and factors associated with MSDs, they may be able to screen for MSDs more effectively. A screening method for MSD in the primary care setting is proposed to facilitate early referral and intervention in order to reduce further 
complications and decrease the overall cost of care [5]. Future studies should aim to validate this screening tool in order to improve early diagnosis and management of these patients.

Acknowledgments: The authors would like to thank Jenna Cornell, DPT (Physical Therapy at St. Luke's, St. Luke's University Health Network), Janak Bahirwani, MD (Gastroenterology, St. Luke's University Health Network), Hussam Tayel, MD (Internal Medicine, St. Luke's University Health Network), and Ruslan Banai, DO (Internal Medicine, St. Luke's University Health Network) for idea development; Jill Stoltzfus, PhD (Department of Research \& Innovation, St. Luke's University Health Network) for statistical analysis; and Neeraj Patel, BS (Lewis Katz School of Medicine at Temple University/St. Luke's University Health Network, Bethlehem, PA) for literature review assistance.

Research funding: None reported.

Author contributions: All authors provided substantial contributions to conception and design, acquisition of data, or analysis and interpretation of data; all authors drafted the article or revised it critically for important intellectual content; C.K. gave final approval of the version of the article to be published; and all authors agree to be accountable for all aspects of the work in ensuring that questions related to the accuracy or integrity of any part of the work are appropriately investigated and resolved.

Competing interests: None reported.

Informed consent: All patients/participants in this study provided verbal informed consent prior to participation.

Ethical approval: This study was reviewed and approved by the St. Luke's University Health Network's Institutional Review Board (protocol SLQI 2017-80).

\section{References}

1. Sahrmann S, Azevedo DC, Dillen LV. Diagnosis and treatment of movement system impairment syndromes. Braz J Phys Ther 2017; 21:9.

2. The American Physical Therapy Association. Physical therapist practice and the human movement system [White paper]. 2015:1-4. https://www.apta.org/contentassets/fadbcf0476484eba9b790c 9567435817/movement-system-white-paper.pdf.

3. Sahrmann SA. The human movement system: our professional identity. Phys Ther 2014;94:1034-42.

4. McClure P, Tevald M, Zarzycki R, Kantak S, Malloy P, Day K, et al. The 4-element movement system model to guide physical therapist education, practice, and movement-related research. Phys Ther 2021;101:1-10.

5. United States Bone and Joint Initiative. The burden of musculoskeletal diseases in the United States (BMUS), 3rd ed.; 2014. Available from: http://www.boneandjointburden.org [Accessed 21 Dec 2017].
6. Hosseini H. Aging and the rising costs of healthcare in the United States: can there be a solution? Ageing Int 2015;40:229-47.

7. Ellis TD, Colón-Semenza C, DeAngelis TR, Thomas CA, Hilaire MS, Earhart GM, et al. Evidence for early and regular physical therapy and exercise in Parkinson's disease. Semin Neurol 2021;41: 189-205.

8. Fritz JM, Childs JD, Wainner RS, Flynn TW. Primary care referral of patients with low back pain to physical therapy: impact on future health care utilization and costs. Spine 2012;37:2114-21.

9. Holme E, Magnusson SP, Becher K, Bieler T, Aagaard P, Kjaer M. The effect of supervised rehabilitation on strength, postural sway, position sense and re-injury risk after acute ankle ligament sprain. Scand J Med Sci Sports 2007;9:104-9.

10. Dufour S, Hondronicols A, Flanigan K. Enhancing pelvic health: optimizing the services provided by primary health care teams in ontario by integrating physiotherapists. Physiother Can 2019;71: 168-75.

11. Harris P, Taylor R, Minor BL, Elliott V, Fernandez M, O’Neal L, et al. The REDCap consortium: builiding an international community of software partners. J Biomed Inf 2019;95. https://doi.org/10. 1016/j.jbi.2019.103208.

12. Monrad SU, Zeller JL, Craig CL, Diponio LA. Musculoskeletal education in US medical schools: lessons from the past and suggestions for the future. Curr Rev Musculoskelet Med 2011;4: 91-8.

13. Sabesan VJ, Schrotenboer A, Habeck J, Lombardo D, Stine S, Jildeh TR, et al. Musculoskeletal education in medical schools: a survey of allopathic and osteopathic medical students. J Am Acad Orthop Surg Glob Res Rev 2018;2:e019.

14. Matzkin E, Smith MEL, Freccero CD, Richardson AB. Adequacy of education in musculoskeletal medicine. JBJS 2005;87:310-4.

15. Cornell J, Kareha S. The prevalence of movement-related conditions in the primary care setting. J Orthop Sports Phys Ther 2018;48:A96-7.

16. CMS. Physician quality reporting system (PQRS) overview. Available from: https://www.cms.gov/files/document/pqrs-overview-factsheet-2014-04-22-remediated.pdf [Accessed 27 Nov 2020].

17. USPSTF. Screening for obesity in children and adolescents: recommendation statement. Am Fam Physician 2017;96:528A-E.

18. Phelan EA, Mahoney JE, Voit JC, Stevens JA. Assessment and management of fall risk in primary care settings. Med Clin North Am 2015;99:281-93.

19. Inserro A. CDC study puts economic burden of asthma at more than $\$ 80$ billion per year. Available from: https://www.ajmc. com/view/cdc-study-puts-economic-burden-of-asthma-at-morethan-80-billion-per-year [Accessed 4 Oct 2021].

20. Choy EHS. The role of sleep in pain and fibromyalgia. Nat Rev Rheumatol 2015;11:513-20.

21. Watenpaugh DE. The role of sleep dysfunction in physical inactivity and its relationship to obesity. Curr Sports Med Rep 2009;8:331-8.

22. Pleyer C, Bittner H, Locke GR, Choung RS, Zinsmeister AR, Schleck $C D$, et al. Overdiagnosis of gastro-esophageal reflux disease and underdiagnosis of functional dyspepsia in a USA community. Neuro Gastroenterol Motil 2014;26:1163-71.

23. Heidelbaugh JJ, Kim AH, Chang R, Walker PC. Overutilization of proton-pump inhibitors: what the clinician needs to know. Therap Adv Gastroenterol 2012;5:219-32.

24. Kirkland EB, Heincelman M, Bishu KG, Schumann SO, Schreiner A, Axon RN, et al. Trends in healthcare expenditures 
among US adults with hypertension: national estimates 20032014. JAHA 2018;7. https://doi.org/10.1161/JAHA.118.008731.

25. CDC. Health and economic costs of chronic diseases. https:// www.cdc.gov/chronicdisease/about/costs/index.htm [Accessed 4 Oct 2021].

26. Lecrubier $Y$. The burden of depression and anxiety in general medicine. J Clin Psychiatr 2001;62:4-9.

27. Harris P, Taylor R, Thielke R, Payne J, Gonzalez N, Conde J. Research electronic data capture (REDCap)- A metadata-driven methodology and workflow process for providing translational research informatics support. J Biomed Inf 2009;42:377-81.

28. Greenland S. Modeling and variable selection in epidemiologic analysis. AM J Public Health 1989;79:340-9.

29. Strauss A, Corbin J. Basics of qualitative research: grounded therory procedures and techniques. Sage Publications; 1990.

30. Kahan SI. Practical strategies for engaging individuals with obesity in primary care. Mayo Clin Proc 2018;93:351-9.

31. CMS. Quality payment program. Available from: https://qpp. cms.gov/mips/explore-measures [Accessed 30 Mar 2021].

32. Childs JD, Fritz JM, Wu SS, Flynn TW, Wainner RS, Robertson EK, et al. Implications of early and guideline adherent physical therapy for low back pain on utilization and costs. BMC Health Serv Res 2015;15:150.

33. Task Force on the Low Back Pain Clinical Practice Guidelines. American osteopathic association guide for osteopathic manipulative treatment (OMT) for patients with low back pain. J Am Osteopath Assoc. 2016;116:536-49.

34. Licciardone JC, Schultz MJ, Amen B. Osteopathic manipulation in the management of chronic pain: current perspectives. J Pain Res 2020;13:1839-47.

35. Cuccia AM, Caradonna C, Annunziata V, Caradonna D. Osteopathic manual therapy versus conventional conservative therapy in the treatment of temporomandibular disorders: a randomized controlled trial. J Bodyw Mov Ther 2010;14:179-84.

36. Steihaug $S$, Alhsen B, Malterud K. From exercise and education to movement and interaction. Treatment groups in primary care for women with chronic muscular pain. Scand J Prim Health Care 2001;10.

37. Chang P, Friedenberg F. Obesity and GERD. Gastroenterol Clin North Am 2014;43:161-73.

38. Byun M, Kim J, Kim M. Physical and psychological factors affecting falls in older patients with arthritis. Int J Environ Res Public Health 2020;17. https://doi.org/10.3390/ ijerph17031098.

39. Sivertsen B, Lallukka T, Petrie KJ, Steingrimsdottir OA, Stubhaug A, Nielsen CS. Sleep and pain sensitivity in adults. Pain 2015;156:1433-9.

40. Chait MC. Gastroesophageal reflux disease: important considerations for the older patients. World J Gastrointest Endosc 2010;2:388-96.

41. Kuchera M, Kuchera W. Osteopathic considerations in systemic dysfunction, Rev 2nd ed. Columbus, OH: Greyden Press LLC; 1994.

42. Lee SP, Sung I, Kim JH, Lee S, Park HS, Shim CS. The effect of emotional stress and depression on the prevalence of digestive diseases. J Neurogastroenterol Motil 2015;21:273-82.

43. Sieck CJ, Hefner JL, Wexler R, Taylor CA, McAlearney AS. Why do they do that? Looking beyond typical reasons for non-urgent ED use among Medicaid patients. Patient Exp J 2016;3:22-30.

44. Durand A, Palazzolo S, Tanti-Hardouin N, Gerbeaux P, Sambuc R, Gentile S. Nonurgent patients in emergency departments: rational or irresponsible consumers? Perceptions of professionals and patients. BMC Res Notes 2012;5. https://doi.org/10.1186/1756-0500-5-525.

45. Delitto A, Piva SR, Moore CG, Fritz JM, Wisniewski SR, Josbeno DA, et al. Surgery versus nonsurgical treatment of lumbar spinal stenosis: a randomized trial. Ann Intern Med 2015;162:465-73.

Supplementary Material: The online version of this article offers supplementary material (https://doi.org/10.1515/jom-2021-0185). 